\title{
A NEW $\mathrm{H}_{2} \mathrm{O}$ ICE HUGONIOT: IMPLICATIONS FOR PLANETARY IMPACT EVENTS
}

\author{
S. T. Stewart ${ }^{1,2}$ and T. J. Ahrens ${ }^{1}$ \\ ${ }^{1}$ Lindhurst Laboratory of Experimental Geophysics, California Institute of Technology, Pasadena, CA 91106 \\ ${ }^{2}$ Present address: Department of Earth and Planetary Sciences, Harvard University, Cambridge, MA 02138
}

\begin{abstract}
Collisions on icy planetary bodies produce impact melt water, redistribute ground ice, and deposit thermal energy available for chemical reactions. The amount of melt generated from an impact is sensitive to the initial temperature, which ranges from the $273 \mathrm{~K}$ on Earth and Mars to $40 \mathrm{~K}$ on the surface of Pluto. Previous shock wave studies, centered at $\sim 263 \mathrm{~K}$ for terrestrial applications, had difficulty defining the onset of phase transformations on the ice Hugoniot, and consequently, the criteria for shock melting was poorly constrained. Because ices on most planetary surfaces exist at ambient temperatures much below $263 \mathrm{~K}$, we conducted a detailed study of the shock response of solid ice at $100 \mathrm{~K}$ and $\sim 40 \%$ porous ice at $\sim 150 \mathrm{~K}$ to derive Hugoniots and critical pressures for shockinduced melting that are applicable to most of the solar system. New Hugoniots for solid and $\sim 40 \%$ porous ice are defined, and the critical pressures required to induce incipient melting and complete melting upon isentropic release from the shock state are revised using calculated shock temperatures and entropy. The critical pressures required for incipient melting of solid ice are only 0.6 and $4.5 \mathrm{GPa}$ for 263 and $100 \mathrm{~K}$ respectively, and pressures between 0.1-0.5 GPa initiate melting of porous ice between 250 and $150 \mathrm{~K}$. Therefore, hypervelocity impact cratering on planetary surfaces, with peak shock pressures $>100 \mathrm{GPa}$, and mutual collisions between porous cometesimals in the outer solar system, with peak pressures of $\sim 1 \mathrm{GPa}$, will generate prodigious amounts of shock-induced melt water.
\end{abstract}

\section{INTRODUCTION}

Throughout the history of the solar system, collisions have played an important role in shaping the topography and modifying the composition of the surfaces of planets and small bodies, such as asteroids and comets. For volatile species such as $\mathrm{H}_{2} \mathrm{O}$ ice, impact processing has produced melt, redistributed deposits, and driven chemical reactions such as dehydration. In the solar system, $\mathrm{H}_{2} \mathrm{O}$ exists over a wide range of temperatures and in a variety of natural settings, including pure crystal density deposits on the surfaces of planets and satellites and intermixed with silicates and organic materials within porous cometesimals.
Previous shock wave studies on solid and porous $\mathrm{H}_{2} \mathrm{O}$ ice, centered just below freezing for terrestrial applications [1,2, and references within], had difficulty characterizing the low-pressure regions on the Hugoniot and, hence, poorly constrained the criteria for shock-induced melting. In addition, the number of $\mathrm{H}_{2} \mathrm{O}$ phases stable at the temperatures found in the outer solar system (Fig. 1) suggests that direct examination of the Hugoniot at low temperatures is necessary to understand the outcome of impact events on icy planetary bodies.

We conducted a study of the shock response of solid ice at $100 \mathrm{~K}$ and $\sim 40 \%$ porous ice at $\sim 150 \mathrm{~K}$ to derive Hugoniots and critical pressures for shock-induced melting that are applicable to most of the solar system. 


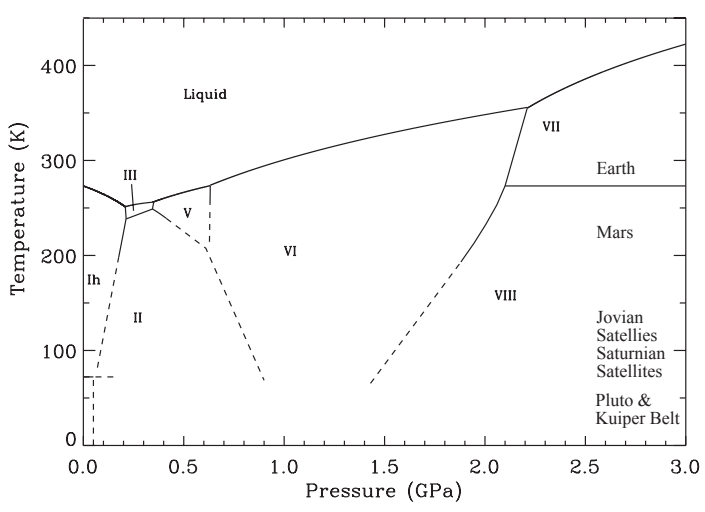

FIGURE 1. Partial $\mathrm{H}_{2} \mathrm{O}$ phase diagram showing only stable phases [3 and references within]. Mean surface temperatures of solar system objects noted on right.
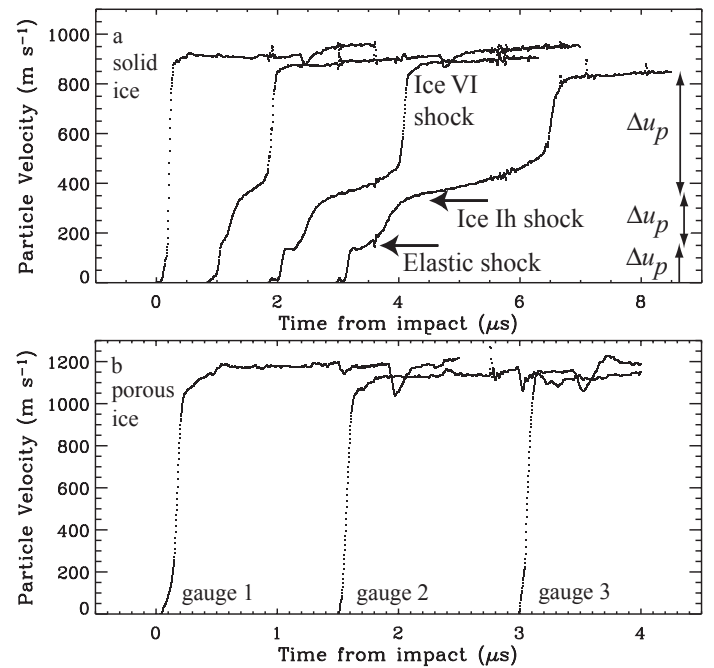

FIGURE 2. a. Particle velocity vs. time gauge records measured at 3-mm intervals within $100 \mathrm{~K}$ solid ice target subject to $1473 \mathrm{~m} \mathrm{~s}^{-1}$ impact by polycarbonate projectile producing 3-wave shock front and $2.1 \mathrm{GPa}$ peak pressure [4]. Reproduced by permission of American Geophysical Union. b. Records from $1572 \mathrm{~m} \mathrm{~s}^{-1}$ impact to $1.4 \mathrm{GPa}$ in $40 \%$ porous ice at $150 \mathrm{~K}$.

\section{EXPERIMENTAL PROCEDURE}

Shock wave profiles, representing Lagrangian particle velocity vs. time (Fig. 2), were integrated to yield shock states (stress and volume) [4-7]. Target assemblies, consisting of 2 thermocouples and 3 or 4 electromagnetic particle velocity gauges embedded at $3-\mathrm{mm}$ intervals between $50-\mathrm{mm}$ diameter ice discs, were hung within a planar magnetic field and cooled by liquid nitrogen spray (Fig. 3). Impact-induced material motion from planar, $40-\mathrm{mm}$ diameter polycarbonate projectiles generated voltage across the gauges, which was recorded by $500 \mathrm{MHz}$ digital oscilloscopes. The measured particle velocities were within $2 \%$ of the impedance-match solution [8] derived from the polycarbonate Hugoniot [9] and impact velocity.

Ice samples and target assemblies were prepared in the Caltech cold room. Solid ice discs were cored from bubble-free carving ice then polished. Porous ice discs were molded from crushed carving ice grains sifted to $180-355 \mu \mathrm{m}$. Bulk densities were determined from the measured mass and volume.

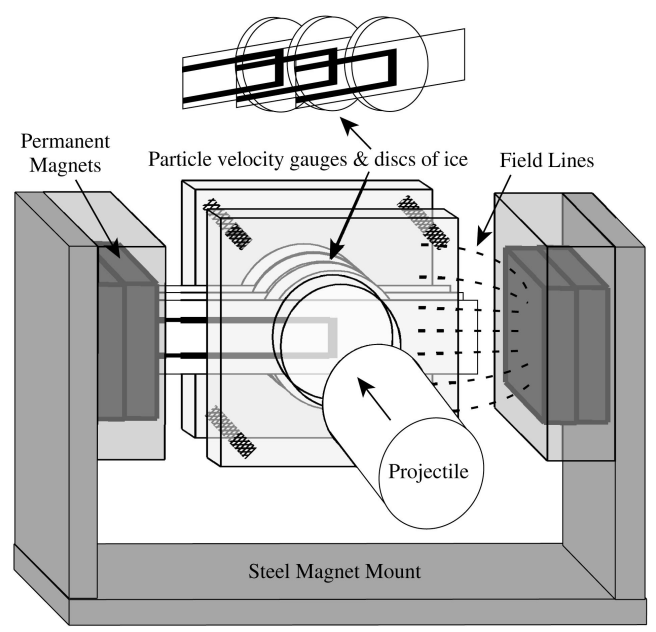

FIGURE 3. Sketch of particle-velocity gauge method for measuring Hugoniot states. $U_{S}$ was determined from wave arrival time at each gauge. $u_{P}$ was calculated from voltage signal, $E$, using Faraday's law, $u_{P}=E /(L H)$, gauge length $L$, and magnetic field strength $H$ [4]. Reproduced by permission of American Geophysical Union.

\section{RESULTS}

\section{Solid Ice Hugoniot}

In the low-temperature solid ice shock data, the onset of phase transformations from the initial ice Ih structure are readily identified from experiment records showing multiple-wave shock fronts. When $T_{0}=100 \mathrm{~K}$, shock compression to the first highpressure phase on the Hugoniot, ice VI, generates 
3-wave shock fronts, consisting of the elastic precursor, ice Ih deformation shock, and ice VI transformation shock (e.g., Fig. 2a). Three-wave profiles are not observed in the $263 \mathrm{~K}$ data [e.g., 7], where shock profiles are composed of the elastic precursor and transformation shock. High-pressure phases are identified by comparing the specific volume in the shock state and calculated shock temperature to the full $P-V-T$ equation of state (EOS) for $\mathrm{H}_{2} \mathrm{O}$.

TABLE 1. Solid ice shock equation of state: $U_{S}=c+s \Delta u_{P}$. For $100 \mathrm{~K}: \rho_{0}=932 \mathrm{~kg} \mathrm{~m}^{-3}[10]$, $\sigma_{E}=0.55 \mathrm{GPa}$, ice Ih cusp at $1.15 \mathrm{GPa}$. For $263 \mathrm{~K}$ : $\rho_{0}=918 \mathrm{~kg} \mathrm{~m}^{-3}, \sigma_{E}=0.2 \mathrm{GPa}$.

\begin{tabular}{lcclrr}
\hline $\begin{array}{c}\text { Hugoniot } \\
\text { Region }\end{array}$ & $c$ & \multicolumn{2}{c}{$s$} & \multicolumn{2}{c}{$\Delta u_{P}\left(\mathbf{m ~ s}^{-1}\right)$} \\
$\left(\mathbf{m ~ s}^{-1}\right)$ & & $\ldots$ & Min & Max \\
\hline 1. Elastic & $3610( \pm 61)$ & 0.92 & $( \pm .63)$ & 0 & 175 \\
2. Ice Ih & $3000( \pm 100)$ & 1.00 & $( \pm .80)$ & 0 & 230 \\
3. Ice VI & $388( \pm 78)$ & 2.61 & $( \pm .14)$ & 100 & 850 \\
4. Ice VII & $1200( \pm 140)$ & 1.46 & $( \pm .11)$ & 600 & 1540 \\
5. Liquid & $1700( \pm 130)$ & 1.440 & $( \pm .035)$ & 1590 & $\ldots$ \\
\hline
\end{tabular}

The present $100 \mathrm{~K}$ and previously published $263 \mathrm{~K}$ solid ice data are combined to derive five distinct regions on the ice Hugoniot: elastic shocks in ice Ih, ice Ih deformation shocks, and shock transformation to ices VI, VII and liquid water [4]. The Hugoniot is expressed as linear fits between shock velocity, $U_{S}$, and the jump in particle velocity, $\Delta u_{p}$, for each wave in the shock front (Table 1). Rather than the more common absolute particle velocity, the change in particle velocity $\Delta u_{p}$ is utilized because the principal stress in the elastic precursor, which varies between 0.2 and $0.55 \mathrm{GPa}$, is comparable to the $0.6 \mathrm{GPa}$ onset of phase transformations on the Hugoniot. To derive the $U_{S}-\Delta u_{p}$ EOS in the multiple-wave region $(<6$ GPa), we include only the published data that record steady shock waves and resolve each wave in the shock front to account for the elastic precursor. We include all published points $>6 \mathrm{GPa}$.

The amplitude of the elastic shock in ice Ih is strongly dependent on the initial temperature and final shock pressure, which is proportional to the strain rate in the shock front [11]. The maximum stress supported before dynamic yielding, $\sigma_{E}$ (GPa), depends on the initial ice temperature, $T_{0}$, and final shock pressure, $P_{S}(\mathrm{GPa})$, by

$$
\begin{aligned}
& \sigma_{E}= \\
& \left\{\begin{array}{l}
0.398( \pm 0.029)+0.039( \pm 0.012) P_{S}, T_{0} \sim 100 \mathrm{~K} \\
0.161( \pm 0.010)+0.035( \pm 0.007) P_{S}, T_{0} \sim 263 \mathrm{~K}
\end{array}\right.
\end{aligned}
$$

The use of $\Delta u_{p}$ removes the temperature variability in the elastic precursor from the shock EOS, and a single set of equations may be used to define both the 100 and $263 \mathrm{~K}$ Hugoniots.

The Hugoniots are constructed in segments using the $U_{S}-\Delta u_{p}$ EOS. First, the elastic shock region is derived from the Rankine-Hugoniot (RH) equations and the zero-pressure volume. Then, the mean elastic shock is used as the initial condition for the RH equations to describe the second region, and so on. The differences between the 100 and 263 K Hugoniots (Fig. 4) arise from: (1) the initial volume dependence on temperature; (2) the temperature-dependent magnitude of the mean elastic shock, $\sigma_{E}$; (3) the presence of an ice Ih transition shock preceding the ice VI transformation shock on the $100 \mathrm{~K}$ curve (forming 3-wave shock profiles.

On the $100 \mathrm{~K}$ Hugoniot, shocks between 0.6 and 2.2 GPa have a three-wave profile, and shocks between 2.2 and $5.5 \mathrm{GPa}$ have two-wave profiles, an elastic shock and ice VII transformation shock. The datum at $7 \mathrm{GPa}$ [12] shows a sudden increase in specific volume along the Hugoniot, indicating the onset of liquid water in the shock state (region 5 , Fig. 4). At shock pressures $>5.5 \mathrm{GPa}$, direct shock transformation to liquid water overdrives the elastic precursor, resulting in a single wave shock front.

\section{Porous Ice Hugoniot}

At $T_{0} \sim 150 \mathrm{~K}$, porous ice samples with bulk density of $0.54 \mathrm{~g} \mathrm{~cm}^{-3}$ were compressed to specific volumes less than or equal to solid ice $\mathrm{Ih}$ at pressures between 0.25 and $1.4 \mathrm{GPa}$ via singlewave shock fronts (e.g., Fig. 2b). The combined $\sim 150 \mathrm{~K}$ (this work) and $\sim 250 \mathrm{~K}$ [13] shock data on $40-45 \%$ porous ice (Fig. 5 , inset) are fit by

$$
U_{S}\left(\mathrm{~m} \mathrm{~s}^{-1}\right)=-50( \pm 110)+1.94( \pm .11) u_{p},
$$


for $u_{p}<2000\left(\mathrm{~m} \mathrm{~s}^{-1}\right)$. Although the $U_{S}-u_{p}$ shock EOS is the same between the two data sets, the shock states and criteria for melting vary slightly because of the different initial temperatures.

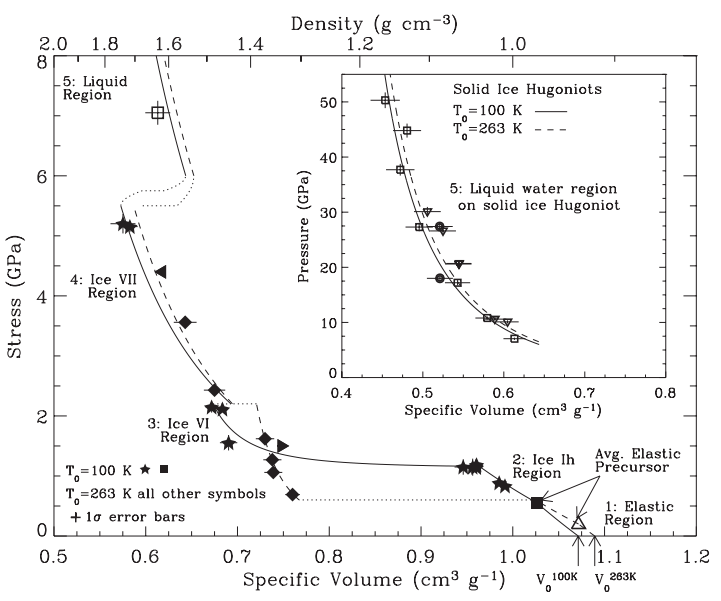

FIGURE 4. Solid ice Hugoniots centered at $100 \mathrm{~K}$ (solid) and $263 \mathrm{~K}$ (dashed) [4]. Dots connect the 5 Hugoniot regions. Data sources: $\square \star$ this work, $\Delta \bullet$ [7], $\square$ $[12], \nabla \bigcirc[14], \diamond[15], \triangleleft[16]$. Reproduced by permission of American Geophysical Union.

The $40-45 \%$ porous ice Hugoniot is multivalued between $0.8-1.1 \mathrm{~cm}^{3} \mathrm{~g}^{-1}$ (Fig. 5) because of the presence of phase transformations, similar to the multi-valued region on the solid ice Hugoniot between $0.55-0.65 \mathrm{~cm}^{3} \mathrm{~g}^{-1}$ (Fig. 4). For $T_{0} \sim 250 \mathrm{~K}$, the first datum, at $0.14 \mathrm{GPa}$, is compressed to a specific volume less than solid ice Ih. This indicates the immediate onset of partial melting along the Hugoniot. When $T_{0} \sim 150 \mathrm{~K}$, however, $40 \%$ porous ice is compressed to solid ice specific volume at $0.26 \mathrm{GPa}$, and partial melt states are generated for shocks above $\sim 0.5 \mathrm{GPa}$.

In both data sets, the shock states reach a minimum volume of $\sim 0.8 \mathrm{~cm}^{3} \mathrm{~g}^{-1}$ in a narrow pressure range near $0.5 \mathrm{GPa}$, where a mixture of liquid and a high-pressure solid phase, e.g. ice $\mathrm{V}$ or VI, may be achieved. The subsequent increase in specific volume with pressure is related to the increasing fraction of liquid in the shock state. Above about $2 \mathrm{GPa}$, the internal energy generated in the shock overcomes the latent heat of fusion, forming pure liquid states on the $\sim 40 \%$ porous ice Hugoniot.

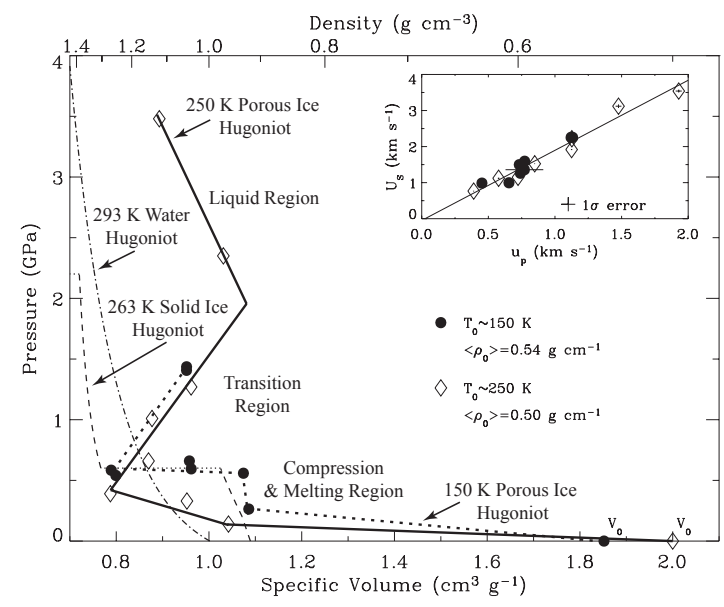

FIGURE 5. 40-45 \% porous ice shock data compared to liquid water and solid ice Hugoniots. Data sources: $\bullet$ this work, $\diamond[13]$.

\section{DISCUSSION}

Using the new $\mathrm{H}_{2} \mathrm{O}$ ice Hugoniots, we derive the critical shock pressures required to initiate and complete shock-induced melting of ice on the solid surfaces of planets and satellites and in porous small bodies such as comets. In solid ice, the shock-induced phase transformation of ice Ih to ices VI and VII, corresponding $>40 \%$ increase in density (Fig. 4), gives rise to large increases in entropy. These lead to shock-induced melting at surprisingly low pressures. In highly distended icy materials, such as found in comets and their precursors, the large $P \Delta V$ irreversible energy deposition during compaction of pore spaces initiates melting at very low shock pressures.

The critical pressures required for incipient (IM) and complete (CM) melting upon release from the shock are determined from the entropy on the Hugoniot. In Fig. 6, we assume that entropy increases only via shock compression and rarefaction unloads the ice isentropically from the Hugoniot state.

In the pressure-entropy diagram, it is immediately apparent that the $263 \mathrm{~K}$ ice Hugoniot enters a mixed solid-liquid phase region upon 
compression above $\sim 0.1 \mathrm{GPa}$. Consequently, the shock temperatures will follow the melting curve (Fig. 1). From 263 K, elastic shock precursors, with principal stresses up to $0.3 \mathrm{GPa}$, metastably drive ice $\mathrm{Ih}$ into the ice $\mathrm{Ih}+\mathrm{L}$ and ice $\mathrm{III}+\mathrm{L}$ stability fields. Above $0.6 \mathrm{GPa}$, upon complete loss of material strength, the $263 \mathrm{~K}$ shock data are in excellent agreement with the equilibrium phase diagram. Shock transformation to ice VI at $0.6 \mathrm{GPa}$ generates the entropy required for incipient melting $\left(2300 \mathrm{~J} \mathrm{~kg}^{-1} \mathrm{~K}^{-1}\right)$, and within the ice VII region, a shock pressure of $3.7 \mathrm{GPa}$ provides sufficient entropy for complete melting $\left(3500 \mathrm{~J} \mathrm{~kg}^{-1} \mathrm{~K}^{-1}\right)$.

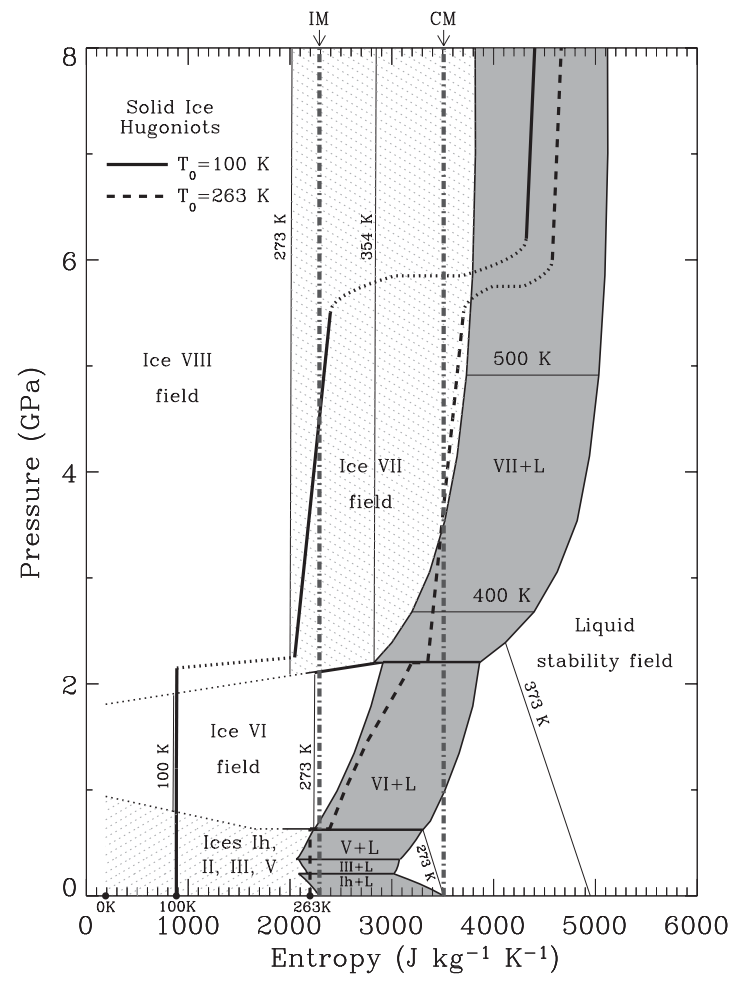

FIGURE 6. 100 and $263 \mathrm{~K}$ ice Hugoniots and partial simplified $\mathrm{H}_{2} \mathrm{O}$ phase diagram in pressure-entropy space [3, 17-20]. IM and CM denote critical entropies for incipient and complete melting upon release to zero pressure. Both Hugoniots exit the ice VII-liquid boundary and enter the liquid field around $20 \mathrm{GPa}$ (above plotted pressure range) [4]. Reproduced by permission of American Geophysical Union.

Unlike at $263 \mathrm{~K}$, there is a negligible increase in entropy as the $100 \mathrm{~K}$ Hugoniot curve crosses from ice Ih to the ice VI field above $0.6 \mathrm{GPa}$ because the elastic shock and ice Ih deformation shock in the 3-wave shock profiles mimic quasiisentropic multiple-shock compression (e.g., Fig. 2b). Above $4.5 \mathrm{GPa}$ on the $100 \mathrm{~K}$ Hugoniot, sufficient entropy is produced for incipient melting upon release, and the entropy for complete melting requires shock pressures $>5.5 \mathrm{GPa}$. Previous estimates of the critical shock pressures for melting solid ice were two to five times higher [21, 22] or limited to $263 \mathrm{~K}$ ice [23].

On the $40-45 \%$ porous ice Hugoniot, the internal energy increase during compaction to full density easily surpasses the latent heat of melting above $\sim 1 \mathrm{GPa}$. All $\sim 250 \mathrm{~K}$ data are denser than the solid ice Hugoniot, indicating partial melting in the shock state and sufficient entropy for incipient melting upon release from shock pressures as low as $0.14 \mathrm{GPa}$. From $\sim 150 \mathrm{~K}$, shock pressures between 0.3 and $0.5 \mathrm{GPa}$ are required for IM. Above $2 \mathrm{GPa}$, pure liquid water is present in the shock state, which requires that the entropy is sufficient for CM upon release (Fig. 6).

For compressions above 5.5 and $\sim 2 \mathrm{GPa}$, solid and $\sim 40 \%$ porous ice Hugoniots may be modeled as "porous" liquid water, respectively $[2,12]$.

\section{CONCLUSIONS}

The new criteria for shock-induced melting of water ice have broad implications for the outcome of collisions in the solar system. The amount of melting is sensitive to the location in the solar system, as both solid and porous $\mathrm{H}_{2} \mathrm{O}$ ice Hugoniots show temperature-dependent behavior in the critical pressure range for the onset of melting upon release $(0.1$ to $5.5 \mathrm{GPa})$. Because the critical pressures for melting are much lower then the peak impact pressures on planetary surfaces (typically $>100 \mathrm{GPa}$ ), melting during impact crater formation will be widespread, softening crater morphologies [e.g., 24] and incorporating liquid water into ejecta blankets [5].

Porous ice Hugoniots are particularly important because satellites and minor planets in the outer solar system accreted via collisions between porous, ice-rich planetesimals. Remnants of the early solar system, which were never accreted into planet-size bodies, are observed today in the two main reservoirs for comets: (i) the Oort cloud, a spherical shell of icy bodies surrounding our Sun 
and the source of long-period comets; and (ii) the Kuiper belt, a disk of cometesimals beyond the orbit of Neptune (30-50 AU) and the provenance of short-period comets. Comets are expected to be mixtures of ices, organic, and silicate materials with $40-80 \%$ porosity $[25,26]$. Mutual collisions between cometesimals before ejection into the Oort cloud, with typical impact velocities of 100's $\mathrm{m} \mathrm{s}^{-1}$ [27], and within the Kuiper belt, at $\sim 1000 \mathrm{~m} \mathrm{~s}^{-1}$ [28], generate peak pressures of order $1 \mathrm{GPa}$. Although solid ice would not begin melting at these impact velocities, porous comets will readily undergo partial melting. Therefore, although comets retain the most pristine material from planetary accretion, they have probably experienced some thermal and chemical processing via partial melting during their collisional evolution.

\section{ACKNOWLEDGEMENTS}

This work was supported by the Planetary Geology and Geophysics program under NASA/Goddard grant number NAG5-10198. We appreciate technical support from M. Long, E. Gelle, and C. McCaughey and thank B. Kamb for use of the Caltech cold laboratory. Contribution \#8976, Division of Geological and Planetary Sciences, California Institute of Technology.

\section{REFERENCES}

1. Gaffney, E. S., "Hugoniot of Water Ice," in Ices in the Solar System, edited by J. Klinger, et al., Kluwer, Boston, 1985, pp. 119-148.

2. Trunin, R. F., et al., High Temp. 37, $702-707$ (1999).

3. Petrenko, V. F. and R. W. Whitworth, Physics of Ice, Oxford U. Press, New York, 1999.

4. Stewart, S. T. and T. J. Ahrens, Geophys. Res. Lett. 30, 1332, doi: 10.1029/2002GL016789 (2003).

5. Stewart, S. T., Collisional Processes involving Icy Bodies in the Solar System, California Institute of Technology, Pasadena, CA, 2002.

6. Fowles, R. and R. F. Williams, J. Appl. Phys. 41, 360-363 (1970).

7. Larson, D. B., J. Glaciol. 30, 235-240 (1984).

8. Ahrens, T. J., "Shock Wave Techniques for Geophysics and Planetary Physics," in Methods of Experimental Physics, edited by C.G. Sammis and T.L. Henyey, 1987, pp. 185-235.
9. Marsh, S. P., ed. LASL Shock Hugoniot Data, U. California Press, Berkeley, 1980.

10. Hobbs, P. V., Ice Physics, Oxford U. Press, London, 1974.

11. Miller, G. H. and T. J. Ahrens, Rev. Modern Phys. 63, 919-948 (1991).

12. Bakanova, A. A., et al., Sov. Phys.-JETP 41, 544548 (1976).

13. Furnish, M. D. and M. B. Boslough, "Measuring Hugoniot, Reshock and Release Properties of Natural Snow and Simulants," Sandia Nat. Lab., Sandia Report SAND92-0985, Albuquerque, NM, February, 1996.

14. Anderson, G. D., "The Equation of State of Ice and Composite Frozen Soil Material," Cold Regions Research and Engineering Laboratory, Research Report 257, Hanover, NH, June, 1968.

15. Gaffney, E. S. and E. A. Smith, "HYDROPLUS Experimental Study of Dry, Saturated, and Frozen Geological Materials," Defense Nuclear Agency, Technical Report DNA-TR-93-74, April, 1994.

16. Davies, F. W. and E. A. Smith, "High Pressure Equation of State Investigation of Rocks," Defense Nuclear Agency, Technical Report DNA-TR-94-1, September, 1994.

17. Dorsey, N. E., ed. Properties of Ordinary WaterSubstance, Reinhold Pub. Corp., New York, 1940.

18. Fei, Y., H.-K. Mao, and R. J. Hemley, J. Chem. Phys. 99, 5369-5373 (1993).

19. Mishima, O., N. Mori, and S. Endo, J. Chem. Phys. 70, 2037-2038 (1979).

20. Bosnjakovic, F., U. Renz, and P. Burow, "Mollier Enthalpy, Entropy-Diagram for Water, Steam and Ice," Hemisphere Publishing Corp. Washington, D.C., 1970.

21. Ahrens, T. J. and J. D. O'Keefe, "Shock Vaporization and the Accretion of the Icy Satellites of Jupiter and Saturn," in Ices in the Solar System, edited by J. Klinger, et al., Kluwer, Boston, 1985, pp. 631-654.

22. Kieffer, S. W. and C. H. Simonds, Rev. Geophys. Space Phys. 18, 143-181 (1980).

23. Pierazzo, E., A. M. Vickery, and H. J. Melosh, Icarus 127, 408-423 (1997).

24. Turtle, E. P. and E. Pierazzo, Science 294, 13261328 (2001).

25. Asphaug, E. and W. Benz, Icarus 121, 225-248 (1996).

26. Farnham, T. L. and A. L. Cochran, Icarus 160, 398418 (2002).

27. Stern, S. A. and P. R. Weissman, Nature 409, 589-591 (2001).

28. Durda, D. D. and S. A. Stern, Icarus 145, 220-229 (2000). 